\title{
Protective effects of Shexiang Tongxin Dropping Pill on pituitrin-induced acute myocardial ischemia in rats
}

\author{
SHAN LIN ${ }^{1,2}$, JIANFENG CHU ${ }^{1,2}$, LING ZHANG $^{1,2}$, DAXIN CHEN $^{1,2}$, FEI XIAO $^{1,2}$,

 \\ ${ }^{1}$ Academy of Integrative Medicine; ${ }^{2}$ Fujian Key Laboratory of Integrative Medicine on Geriatrics, \\ Fujian University of Traditional Chinese Medicine, Fuzhou, Fujian 350122, P.R. China; \\ ${ }^{3}$ Rainbow Babies and Children's Hospital, Case Western Reserve University School of Medicine, \\ Cleveland, OH 44106, USA; ${ }^{4}$ Inner Mongolia Conba Pharmaceutical Co., Ltd., Shanghai 201318, P.R. China
}

Received August 31, 2016; Accepted May 16, 2017

DOI: $10.3892 / \mathrm{mmr} .2017 .6963$

\begin{abstract}
Shexiang Tongxin Dropping Pill (STP) is an established traditional Chinese medicine that is widely used for the treatment of ischemic heart disease (IHD), although its mechanisms remain unclear. The present study investigated the protective effects of STP following pituitrin (PTT)-induced myocardial ischemia in rats. ST-segment elevation, blood rheology, and the serum levels of creatine kinase-MB (CK-MB) and lactate dehydrogenase (LDH) were measured. Following heart excision, histological analysis using hematoxylin and eosin and terminal deoxynucleotidyl transferase dUTP nick end labeling were performed. The mRNA expression levels of B-cell lymphoma 2 (Bcl-2) and $\mathrm{Bcl}-2$-associated $\mathrm{X}$ protein (Bax) were determined using reverse transcription-quantitative polymerase chain reaction, and their protein expression was detected using immunohistochemistry. The results demonstrated that STP treatment protected against ST elevation, lowered whole blood viscosity, and reduced the serum levels of CK-MB and LDH following acute myocardial ischemia. In addition, STP treatment restored the histopathological change following PTT-induced myocardial ischemia, and resulted in downregulated expression of Bax and upregulated expression of Bcl-2 in myocardial tissue. The present study demonstrates the cardioprotective ability of STP in a rat model of myocardial ischemic injury, which may be attributed to its anti-apoptotic properties. The cardioprotective properties of STP require further investigation to determine whether it may be used for the clinical treatment of IHDs.
\end{abstract}

Correspondence to: Professor Jun Peng, Academy of Integrative Medicine, Fujian University of Traditional Chinese Medicine, 1 Qiuyang Road, Fuzhou, Fujian 350122, P.R. China

E-mail: pjunlab@hotmail.com

Key words: myocardial ischemia, apoptosis, Shexiang Tongxin Dropping Pill, pituitrin

\section{Introduction}

Myocardial ischemia is a result of impaired coronary blood flow to the heart, which may be caused by various factors, including atherosclerosis, thrombosis or vascular spasm. Ischemia causes the demand for oxygen and the amount of oxygen supplied to the heart to become imbalanced, and this imbalance may lead to damage and necrosis in cardiac tissues. Coronary heart diseases and ischemic heart diseases (IHDs), particularly acute myocardial infarction, are among the leading causes of morbidity and mortality worldwide. According to the World Health Organization, cardiovascular disease is set to become the leading cause of death globally $(1,2)$. Increasing evidence indicates that cardiomyocyte apoptosis occurs during the first few $\mathrm{h}$ of myocardial ischemia, and this leads to widespread myocardial cell damage, cardiac dysfunction and ultimately heart failure. Despite recent advances in research and clinical improvements to combat myocardial ischemia, there have been limited or no fundamental breakthroughs in drug treatment $(3,4)$.

Shexiang Tongxin Dropping Pill (STP) is a traditional China Food and Drug Administration (CFDA)-approved prescription medicine (approval no. Z20080018), which consists of a combination of seven medicinal components, including moschus, Radix et Rhizoma Ginseng, Calculus Bovis, bear gall, Venenum Bufonis, borneol and salvia miltiorrhiza $(5,6)$. In a previous study, we analyzed and identified the major constituents of STP by high-performance liquid chromatography-quadrupole time-of-flight mass spectrometry, and these constituents included triterpene saponins, bufadienolides, bile acids and phenyl allyl compounds. Of the identified constituents, 13 were subsequently quantified by ultra-performance liquid chromatography-triple-quadrupole tandem mass spectrometry (5). STP has been widely used for the clinical treatment of cardiovascular diseases, particularly coronary heart disease in China and Southeast Asia (7-10). A previous pharmacological study indicated that STP may protect endothelial cells against atherosclerotic lesions by reducing the expression of endothelin-1, C-reactive protein and tumor necrosis factor- $\alpha$, while increasing nitrogen oxide levels in the blood (11). A separate study demonstrated that STP attenuates 
atherosclerotic lesions in ApoE deficient mice, indicating potential effects on various pathological, biochemical and molecular aspects of atherosclerosis, including lipid regulation, fibrosis, inflammation and oxidative stress (6). However, the cardioprotective effects of STP on myocardial ischemia and its underlying mechanisms remain largely unknown. The present study investigated the cardioprotective effects of STP in a pituitrin (PTT)-induced rat model of acute myocardial ischemic injury. In addition, the mechanism of action of STP was investigated in order to provide a theoretical basis for the clinical treatment of IHDs.

\section{Materials and methods}

Materials. The present study was approved by the Center of Laboratory Animals, Fujian University of Traditional Chinese Medicine [Fuzhou, China; certified no. SYXK (Min) 2009-0001]. Healthy male Sprague-Dawley rats $(\mathrm{n}=30$; age, $\sim 10$ weeks; weight, 200-220 g) were purchased from Shanghai Laboratory Animal Center Laboratory Animal Co., Ltd. (Shanghai, China). The animals were kept in a temperature-controlled room at $20.1-23.1^{\circ} \mathrm{C}$ and $40-50 \%$ humidity, under a 12-h light/dark cycle and free access to food and water.

STP was provided by Inner Mongolia Conba Pharmaceutical Co., Ltd. (Shanghai, China). Sodium pentobarbital was purchased from Merck KGaA (Darmstadt, Germany). PTT injection was purchased from Ningbo Second Hormone Factory (Ningbo, China). Isosorbide mononitrate (IM) was purchased from Xi'an Lijun Pharmaceutical Co., Ltd. (Xi'an, China). Antibodies against B-cell lymphoma-2 (Bcl-2; cat no. ab32124), Bcl-2-associated X protein (Bax; cat no. ab32503) and GAPDH (cat no. ab9485) were obtained from Abcam (Cambridge, UK). Unless indicated otherwise, all chemicals used were purchased from Sigma-Aldrich (Merck $\mathrm{KGaA})$.

Rat model of acute myocardial ischemia. The rats were randomly assigned into five groups, each containing six rats. Rats in the control group and experimental model group received saline pretreatment. Rats in the STP-low and -high dose groups were pretreated with STP (20 and $40 \mathrm{mg} / \mathrm{kg}$, respectively). Rats in the IM group were pretreated with IM $(4 \mathrm{mg} / \mathrm{kg})$ as the positive control. All pretreatments involved oral administration for seven days, at a volume of $5 \mathrm{ml} / \mathrm{kg} /$ day.

Myocardial ischemia was established by PTT injection as previously described (12-14). Briefly, after $1 \mathrm{~h}$ of the final pretreatment, the rats were anesthetized with sodium pentobarbital (30 mg/kg injected intraperitoneally). Rats then received PTT by subcutaneous injection $(40 \mathrm{U} / \mathrm{kg}$, except for the control group) to record electrocardiosignals immediately. After $30 \mathrm{~min}, 8-\mathrm{ml}$ blood samples were collected from the abdominal aorta and separated for serum enzyme assays. Hearts were excised, rinsed in ice-cold isotonic saline and blotted with filters for biochemical assays. Blood and heart samples were stored at $-80^{\circ} \mathrm{C}$ prior to further analysis.

Determination of ST-segment elevation. Normal electrocardiograms were recorded by the placement of subcutaneous electrodes connected to an electrocardiograph (BL-420S;
Chengdu Taimeng Software Co., Ltd., Chengdu, China). Electrocardiograms (ECGs) recorded ST-segment elevation immediately following PTT injection.

Determination of blood rheology. The viscosity of whole blood ( $\mathrm{mPa} \cdot \mathrm{s})$ was determined at different shear rates (SRs) in reciprocal sec $\left(10,60\right.$ and $\left.150 \mathrm{~s}^{-1}\right)$ using an automatic blood viscosity meter (LBY-N6B; Beijing Precil Instrument Co., Ltd., Beijing, China).

Determination of creatine kinase- $M B(C K-M B)$ and lactate dehydrogenase $(L D H)$ in the serum. The serum was obtained from whole blood by centrifugation at $1,200 \mathrm{x} \mathrm{g}$ at $4^{\circ} \mathrm{C}$ for $20 \mathrm{~min}$. The serum levels of LDH (cat no. F16113) and CK-MB (cat no. F15213) in the rat blood were measured by the corresponding rat ELISA kits (Shanghai Westang Bio-Tech Co., Ltd., Shanghai, China).

Histological examination of myocardium using hematoxylin and eosin staining. Heart sections were excised and fixed in $4 \%$ paraformaldehyde solution for $24 \mathrm{~h}$ at room temperature. Heart tissues were then processed for paraffin-embedding, sectioning and staining by standard histological methods. The tissues were cut into $5 \mu \mathrm{m}$ sections using a rotary microtome (RM 2235; Leica Microsystems GmbH, Wetzlar, Germany). Slides were stained with hematoxylin for $20 \mathrm{~min}$ and eosin for $2 \mathrm{~min}$ at room temperature and observed under a light microscope (Nikon Corporation, Tokyo, Japan) at x200 magnification.

Determination of myocardial apoptosis by terminal deoxynucleotidyl transferase (TdT) dUTP nick end labeling (TUNEL). Heart sections were excised and fixed in $4 \%$ paraformaldehyde solution for $24 \mathrm{~h}$ at room temperature. Heart tissues were then processed for paraffin-embedding, sectioning and staining by standard histological methods. The tissues were cut into $5 \mu \mathrm{m}$ sections using a rotary microtome. Myocardial apoptosis of the tissues sections was analyzed by TUNEL assay using the TdT in situ apoptosis detection kit (R\&D Systems, Inc., Minneapolis, MN, USA), according to the manufacturer's protocol. Individual nuclei were visualized at a magnification of x400 under a fluorescent microscope (Leica Microsystems $\mathrm{GmbH}$ ) for quantitative analysis. TUNEL-positive cells exhibited apoptosis characteristics, including condensed chromatin and cellular shrinkage. Apoptotic cells were counted as 3,3'-diaminobenzidine (DAB)-positive cells (stained brown) in five randomly selected fields for each slide. The percentage of apoptotic cells was calculated as the ratio of the number of TUNEL-positive cells to the total number of cells, as the mean of the five randomly selected fields.

Reverse transcription-quantitative polymerase chain reaction $(R T-q P C R)$. Total RNA from heart samples was extracted using TRIzol reagent according to the manufacturer's protocol (Invitrogen; Thermo Fisher Scientific, Inc.). Oligo (dT)-primed (Thermo Fisher Scientific, Inc.) RNA $(1 \mu \mathrm{g})$ was reverse transcribed with the PrimerScript RT reagent Kit with gDNA Eraser (cat. no. RR047; Takara Bio, Inc., Otsu, Japan), according to the manufacturer's protocol. The temperature protocol used was as follows: At $37^{\circ} \mathrm{C}$ for $15 \mathrm{~min}$, at $85^{\circ} \mathrm{C}$ for $5 \mathrm{sec}$, followed 
by annealing at $4^{\circ} \mathrm{C}$ for $15 \mathrm{~min}$. cDNA was used to determine the expression of Bcl-2, Bax and GAPDH mRNA using SYBR Green Master Mix (Thermo Fisher Scientific, Inc., Waltham, MA, USA) and the 7500 Fast Real Time PCR System (Applied Biosystems; Thermo Fisher Scientific, Inc.). GAPDH was used as the housekeeping gene was used to normalize the expression of Bcl-2 and Bax. The primers used in the present study were as follows: Bax, forward 5'-GCTGATGGCAACTTC AACTGGG-3', reverse 5'-TTCTTCCAGATGGTGAGCGAG G-3'; Bcl-2, forward 5'-TACCGTCGTGACTTCGCAGAG AT-3', reverse 5'-AGGAGAAATCAAACAGAGGTCGC-3'; and GAPDH, forward 5'-CTGCCTTCTCTTGTGACA-3' and reverse 5'-TGTAGACCATGTAGTTGAGG-3'. The thermocycling conditions were as follows: Pre-denaturation at $95^{\circ} \mathrm{C}$ for $30 \mathrm{sec}$, followed by 40 cycles of denaturation at $95^{\circ} \mathrm{C}$ for $3 \mathrm{sec}$, annealing at $60^{\circ} \mathrm{C}$ for $30 \mathrm{sec}$ and extension at $72^{\circ} \mathrm{C}$ for $15 \mathrm{sec}$. Relative gene expression was calculated according to the $2^{-\Delta \Delta \mathrm{Cq}}$ method (15) and were presented as the fold change compared with the control. The experiment was repeated three times.

Immunohistochemistry (IHC). Myocardial tissues were fixed with $4 \%$ paraformaldehyde for $24 \mathrm{~h}$ at room temperature, and the samples were then processed for paraffin-embedding and sectioning using standard histological methods. The tissues were cut into $5 \mu \mathrm{m}$ sections using a rotary microtome. The slides were rehydrated in gradient ethanol (100, 95, 90, 80 and $70 \%$ ) and distilled water. Subsequently, the slides were subjected to antigen retrieval at $37^{\circ} \mathrm{C}$ for $10 \mathrm{~min}$ and washed with PBS. The endogenous peroxidase activity was quenched using 3\% hydrogen peroxide at room temperature for $10 \mathrm{~min}$. Following blocking of non-specific binding with $5 \%$ bovine serum albumin (Thermo Fisher Scientific, Inc.) in PBS containing $0.1 \%$ Tween-20 at room temperature for $20 \mathrm{~min}$, the slides were incubated with rabbit polyclonal antibodies against Bcl-2 (1:200), Bax (1:200) and GAPDH (1:300) at $37^{\circ} \mathrm{C}$ overnight. After washing with PBS, slides were incubated with biotinylated secondary antibody (1:400; cat no. sc-516142; Santa Cruz Biotechnology, Inc., Dallas, TX, USA) for $20 \mathrm{~min}$ at $37^{\circ} \mathrm{C}$, followed by incubation with $1 \mathrm{mg} / \mathrm{ml}$ horseradish peroxidase (HRP)-labeled streptavidin (cat no. ab7403; Abcam) for $20 \mathrm{~min}$ at $37^{\circ} \mathrm{C}$, and then washed with PBS. The slides were subsequently incubated with DAB as the chromogen for $20 \mathrm{~min}$ at room temperature, followed by counterstaining with diluted Harris hematoxylin (1:10) for $2 \mathrm{~min}$ at room temperature. Following staining, five high-power fields (x400) were randomly selected from each slide under a light microscope (Nikon Corporation, Tokyo, Japan). The mean number of positive cells in each field was counted using Image-Pro Plus software version 6.0 (Media Cybernetics, Inc., Rockville, MD, USA). To rule out any non-specific staining, PBS was used to replace the primary antibody as a negative control.

Statistical analysis. All experiments were repeated at least three times. Data are presented as the mean \pm standard deviation. One-way analysis of variance followed by Dunnett's post hoc test was used for statistical comparisons. Analyses were conducted with SPSS version 18.0 statistical software (SPSS, Inc., Chicago, IL, USA). $\mathrm{P}<0.05$ was considered to indicate a statistically significant difference.
Table I. Variations in hemorheology between groups.

\begin{tabular}{lccc}
\hline & \multicolumn{3}{c}{ Viscosity at different SRs, mPas's } \\
\cline { 2 - 4 } Groups & SR $10 \mathrm{~s}^{-1}$ & SR $60 \mathrm{~s}^{-1}$ & SR $150 \mathrm{~s}^{-1}$ \\
\hline Control & $8.78 \pm 0.75$ & $4.93 \pm 0.24$ & $3.89 \pm 0.17$ \\
Model & $12.09 \pm 0.89^{\mathrm{a}}$ & $5.91 \pm 0.22^{\mathrm{a}}$ & $4.53 \pm 0.24^{\mathrm{a}}$ \\
IM & $11.05 \pm 0.46^{\mathrm{b}}$ & $5.39 \pm 0.61^{\mathrm{b}}$ & $4.24 \pm 0.20^{\mathrm{b}}$ \\
STP-low & $10.73 \pm 1.30^{\mathrm{b}}$ & $5.55 \pm 0.50$ & $4.18 \pm 0.19^{\mathrm{c}}$ \\
STP-high & $10.22 \pm 0.72^{\mathrm{c}}$ & $5.16 \pm 0.18^{\mathrm{c}}$ & $3.94 \pm 0.20^{\mathrm{c}}$ \\
\hline
\end{tabular}

Data are presented as the mean \pm standard deviation, $\mathrm{n}=6 .{ }^{\mathrm{a}} \mathrm{P}<0.01$ vs. control group; ${ }^{b} \mathrm{P}<0.05$ and ${ }^{\mathrm{c}} \mathrm{P}<0.01$ vs. model group. $\mathrm{SR}$, shear rate; IM, isosorbide mononitrate; STP, Shexiang Tongxin Dropping Pill; STP-low, 20 mg/kg STP group; STP-high, 40 mg/kg STP group.

\section{Results}

Effect of STP on ST-segment elevation. Electrocardiograms indicating ST segment elevation were used to demonstrate successful myocardial ischemia and establish the experimental model. At 1 min after PTT administration, ST-segment elevation remained evident in the experimental model group, whereas mice pretreated with STP (20 and $40 \mathrm{mg} / \mathrm{kg}$ dose groups) exhibited significantly reduced ST-segment elevation compared with the model group. The results for the STP-low and high dose groups were similar to those in the IM positive control group (Fig. 1).

Effects of STP on hemorheology. The whole blood viscosities at different SRs in the experimental model group were significantly higher compared with the control group $(\mathrm{P}<0.01)$. However, whole blood viscosities in the STP-low and -high dose treatment groups (20 and $40 \mathrm{mg} / \mathrm{kg}$, respectively) exhibited significant dose-dependent reductions at different SRs compared with the experimental model group. Similarly, the IM group also exhibited significantly reduced whole blood viscosities compared with the experimental model group (Table I). Notably, the whole blood viscosities in the STP-high dose group were lower compared with the IM and STP-low dose groups (Table I).

Effect of STP on CK-MB and LDH serum levels. Levels of the myocardial injury marker enzymes CK-MB and LDH were significantly increased in the experimental model group compared with the control group ( $\mathrm{P}<0.05$; Fig. 2). Pretreatment with STP significantly reduced serum levels of $\mathrm{LDH}$ in a dose-dependent manner compared with the experimental model group ( $\mathrm{P}<0.05$; Fig. 2). Levels of CK-MB were also reduced by pretreatment with STP compared with the experimental model group, however, this reduction was only significant in the high dose group $(\mathrm{P}<0.05$; Fig. 2$)$.

Effect of STP on myocardial histology. Histological analysis demonstrated that the myocardium of control group rats displayed a normal myofibrillar structure with striations, branched appearance and continuity with adjacent myofibrils. 
A

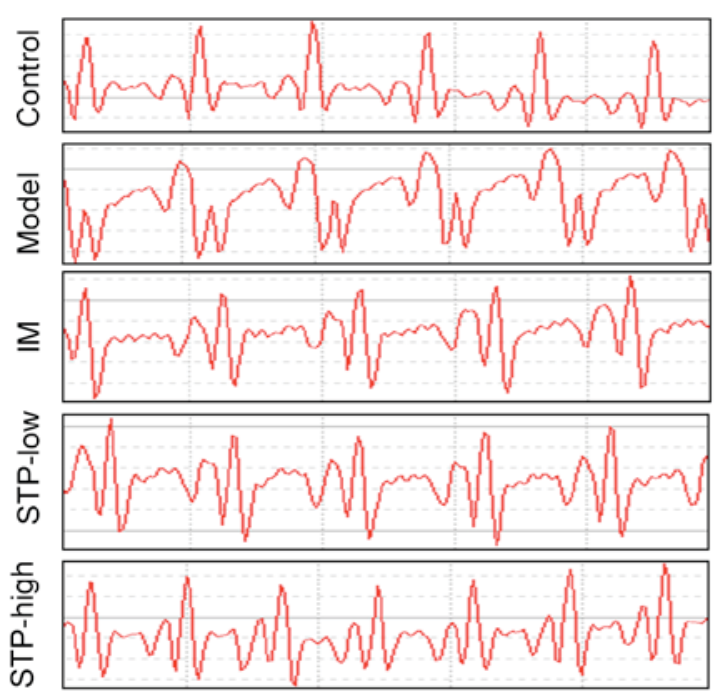

B

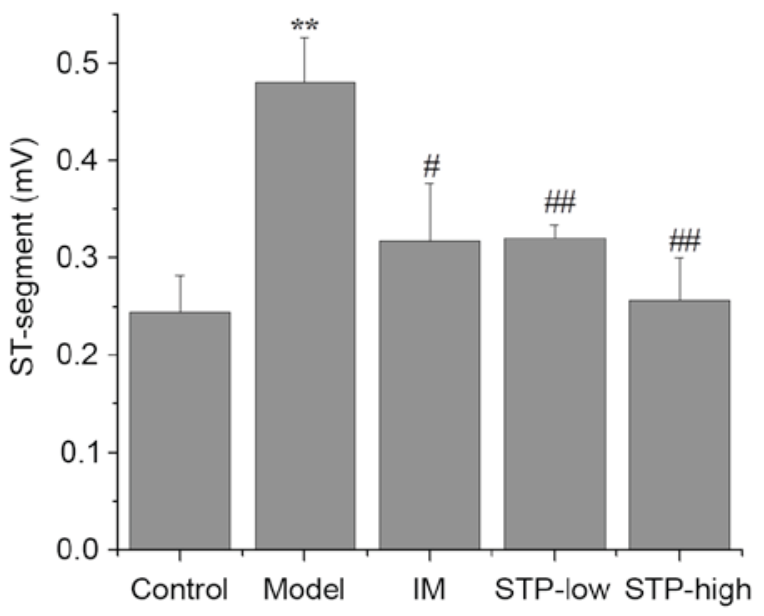

Figure 1. Effects of STP on ECG in PTT-induced acute myocardial ischemia in rats. (A) ECG changes at 1 min after PTT administration. ECG was recorded by the placement of subcutaneous electrodes connected to an electrocardiograph immediately following PTT injection. (B) The average values of ST-segment elevation based on the ECG of each group. Data are presented as the mean + standard deviation, $\mathrm{n}=6$ per group. ${ }^{* *} \mathrm{P}<0.01$ vs. control group; ${ }^{*} \mathrm{P}<0.05$ and ${ }^{\# \#} \mathrm{P}<0.01$ vs. model group. STP, Shexiang Tongxin Dropping Pill; ECG, electrocardiogram; PTT, pituitrin; IM, isosorbide mononitrate; STP-low, 20 mg/kg STP group; STP-high, $40 \mathrm{mg} / \mathrm{kg}$ STP group.

A

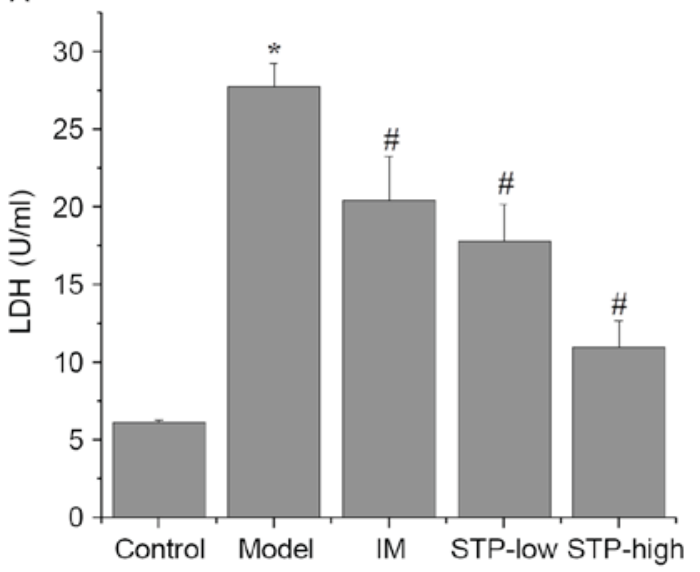

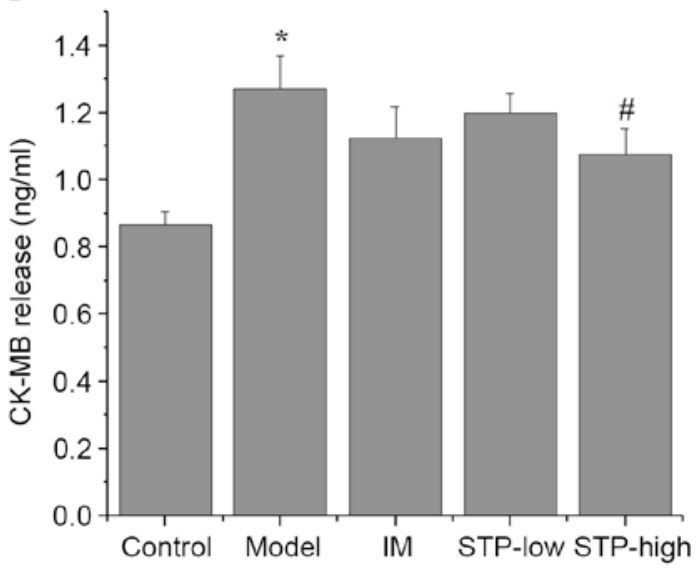

Figure 2. Effects of STP on CK-MB and LDH levels in pituitrin-induced acute myocardial ischemia in rats. Serum levels of (A) LDH and (B) CK-MB in each group. Serum levels were measured with corresponding rat ELISA kits. Data are presented as the mean + standard deviation, $\mathrm{n}=6$ per group. "P $<0.05$ vs. control group; " $\mathrm{P}<0.05$ vs. model group. STP, Shexiang Tongxin Dropping Pill; CK-MB, creatine kinase-MB; LDH, lactate dehydrogenase; IM, isosorbide mononitrate; STP-low, $20 \mathrm{mg} / \mathrm{kg}$ STP group; STP-high, $40 \mathrm{mg} / \mathrm{kg}$ STP group.

By contrast, tissues from the experimental model rats revealed obvious swelling of myocardial cells, degeneration and loss of transverse striations. Tissues from both STP treatment groups exhibited normal, well-preserved myocardial cell morphology. Tissue sections from the IM group also revealed normal myofibrillar structure with obvious transverse striations. These results indicate that STP treatment may protect against tissue necrosis following myocardial ischemia (Fig. 3).

Effect of STP on myocardial apoptosis using TUNEL staining. A significantly higher percentage of TUNEL-positive cells were observed in the experimental model group compared with the control group $(\mathrm{P}<0.05$; Fig. 4). However, pretreatment with low and high doses of STP significantly reduced the percentage of TUNEL-positive cells, compared with the experimental model group $(\mathrm{P}<0.05$; Fig. 4$)$. The IM group also exhibited a significant decrease in the percentage of TUNEL-positive cells compared with the experimental model group ( $\mathrm{P}<0.05$; Fig. 4).

Effect of STP on Bcl-2 and Bax mRNA expression in cardiomyocytes using $R T-q P C R$. There was a significant decrease in Bcl-2 mRNA expression and a significant increase in Bax mRNA expression in the experimental model group, compared with the control group ( $\mathrm{P}<0.05$; Fig. 5$)$. By contrast, STP pretreatment groups exhibited significantly increased Bcl-2 expression and decreased Bax expression, compared with the experimental model group $(\mathrm{P}<0.05$; Fig. 5). In particular, STP pretreatment progressively increased the expression of Bcl-2 in a dose-dependent manner (Fig. 5A), 


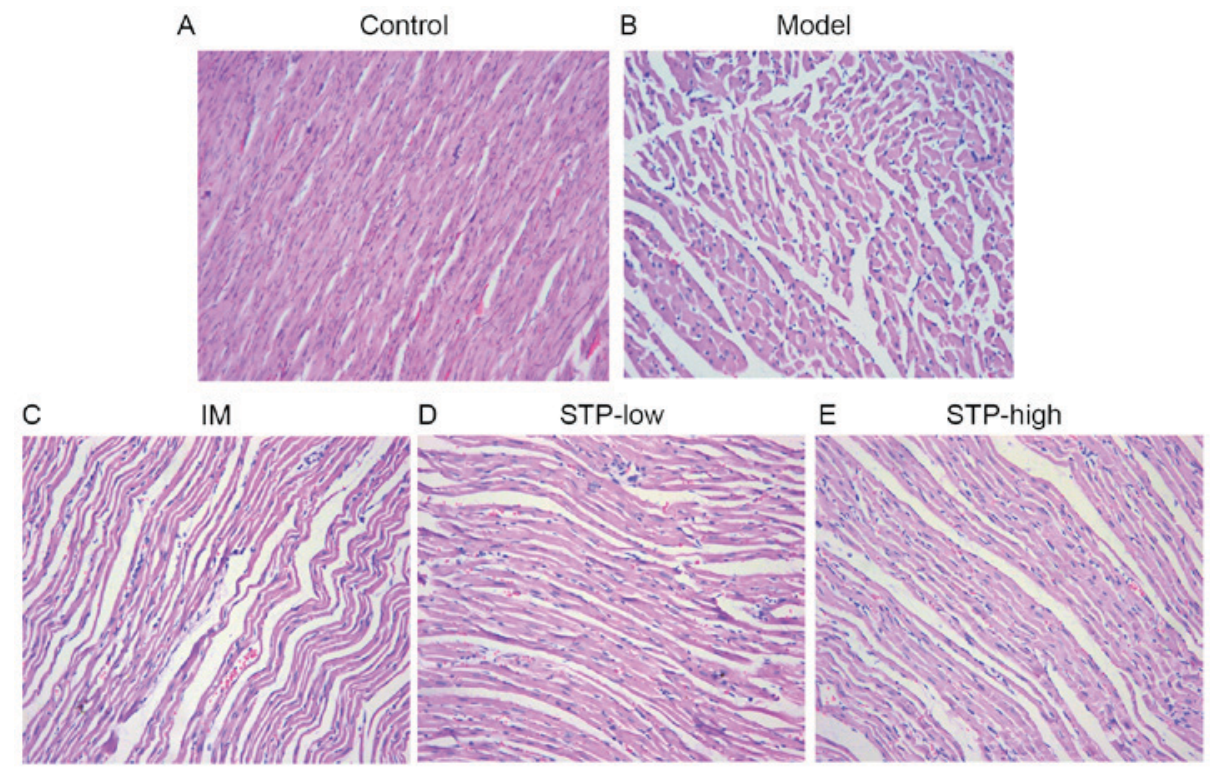

Figure 3. Effects of STP on pathological changes in pituitrin-induced acute myocardial ischemia rat myocardial tissue by hematoxylin and eosin staining. (A) Control group indicating normal myocardial morphology and clear transverse striations. (B) Model group with obvious swelling of myocardial cells, degeneration and loss of transverse striations. (C) IM group with normal myocardial arrangement and clear transverse striations. (D) STP-low exhibited slight myocardial cell swelling, degeneration and unclear horizontal striations. (E) STP-high exhibited diminished myocardial cell swelling and unclear horizontal striations. $\mathrm{n}=6$ per group. Magnification, x200. STP, Shexiang Tongxin Dropping Pill; IM, isosorbide mononitrate; STP-low, 20 mg/kg STP group; STP-high, $40 \mathrm{mg} / \mathrm{kg}$ STP group.

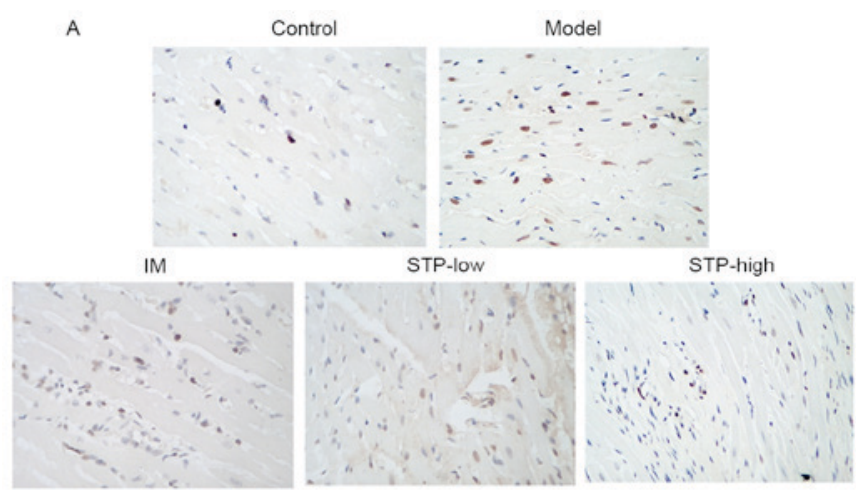

B

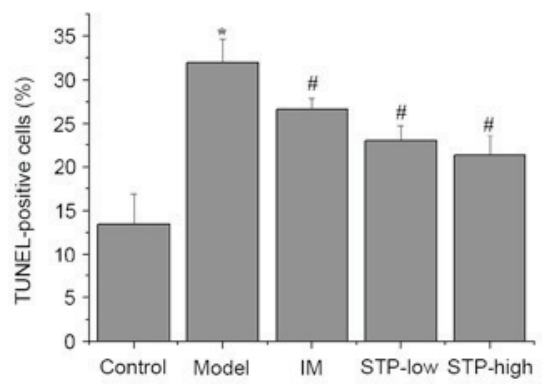

Figure 4. TUNEL staining and morphological analysis of apoptotic damage in pituitrin-induced rat cardiomyocytes. (A) Myocardial tissues were processed for TUNEL staining for apoptosis. (B) Apoptotic cells were characterized by nuclear condensation and dark brown staining, and were visualized under a light microscope. Images were captured at a magnification of $x 400$. Data are presented as the mean + standard deviation, $n=6$ per group. ${ }^{*} \mathrm{P}<0.05$ vs. control group; ${ }^{\#} \mathrm{P}<0.05$ vs. model group. IM, isosorbide mononitrate; STP, Shexiang Tongxin Dropping Pill; STP-low, 20 mg/kg STP group; STP-high, $40 \mathrm{mg} / \mathrm{kg}$ STP group.

whilst the same dose-dependent effect was not observed for Bax expression (Fig. 5B).
Effect of STP on Bcl-2 and Bax protein expression in cardiomyocytes using IHC. There was a significant decrease in the level of cytoplasmic Bcl-2 protein in the experimental model group compared with the control group $(\mathrm{P}<0.01$; Fig. 6). However, STP pretreatment groups exhibited significantly increased Bcl-2 protein expression levels compared with the experimental model group, in a dose-dependent manner $(\mathrm{P}<0.01$; Fig. 6). In addition, the protein expression of Bax in the experimental model group was significantly higher compared with the control group $(\mathrm{P}<0.05$; Fig. 7$)$, and STP pretreatment groups exhibited significantly decreased Bax expression compared with the experimental model group $(\mathrm{P}<0.01$; Fig. 7).

\section{Discussion}

PTT is an extract of hormones that are released from the posterior lobe of the pituitary gland, which consists of vasopressin and oxytocin hormones. These hormones are synthesized in the neuronal cells of hypothalamic nuclei and stored in nerve cell endings in the posterior pituitary gland (16). In 1960, Black (17) used PTT to successfully establish an animal model of acute myocardial ischemia, which was subsequently used in numerous studies investigating myocardial ischemic antagonists $(13,16)$.

The present study demonstrated that STP pretreatment reduced ST-segment elevation, whole blood viscosity, and the mRNA expression of CK-MB and LDH in rats with PTT-induced acute myocardial ischemic injury. These results indicate that STP may have cardioprotective effects that alleviate myocardial ischemic injury.

Electrocardiograms indicating ST-segment elevation were used to demonstrate that myocardial ischemia was successfully established. Observing alterations in the ECG pattern, 
A

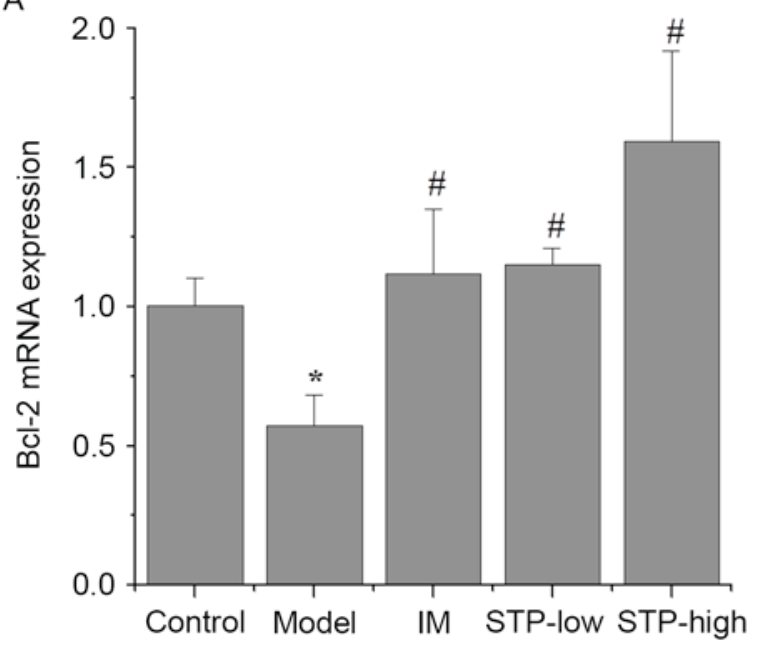

B

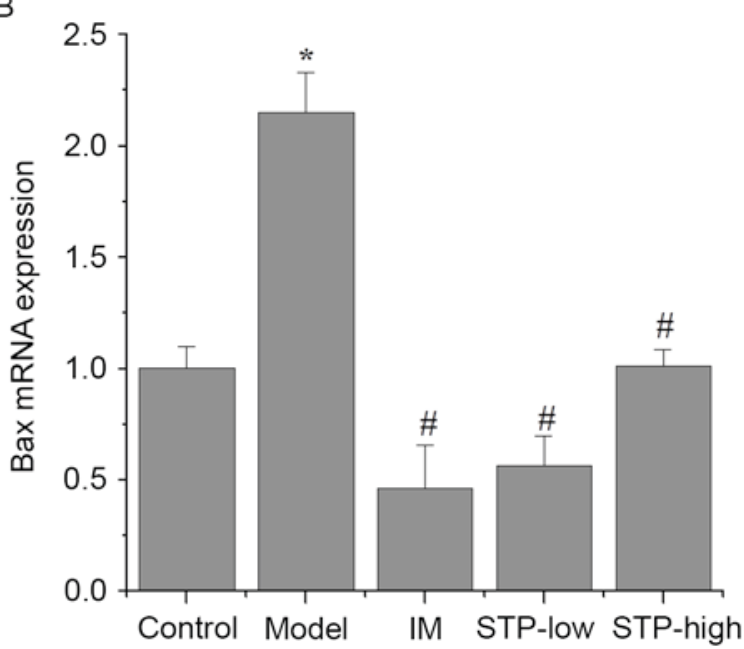

Figure 5. Effects of STP on the mRNA expression of Bcl-2 and Bax in pituitrin-induced acute myocardial ischemia in rat cardiomyocytes. Expression levels of (A) Bcl-2 and (B) Bax mRNA in each group. mRNA expression was determined by reverse transcription-quantitative polymerase chain reaction analysis using the $2^{-\triangle \Delta C q}$ method and expression was presented as the fold change compared with the control. Data are presented as the mean + standard deviation, $n=6$ per group. * $\mathrm{P}<0.05$ vs. control group; ${ }^{\#} \mathrm{P}<0.05$ vs. model group. STP, Shexiang Tongxin Dropping Pill; Bax, Bcl-2-associated X; IM, isosorbide mononitrate; STP-low, $20 \mathrm{mg} / \mathrm{kg}$ STP group; STP-high, $40 \mathrm{mg} / \mathrm{kg}$ STP group.

A

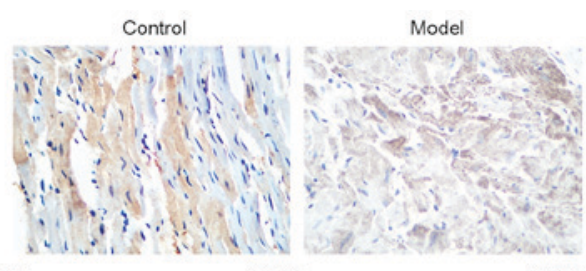

IM

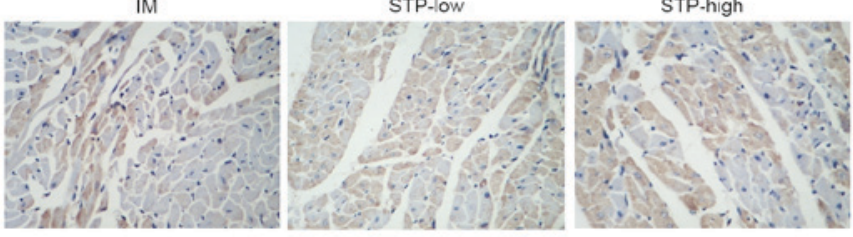

B

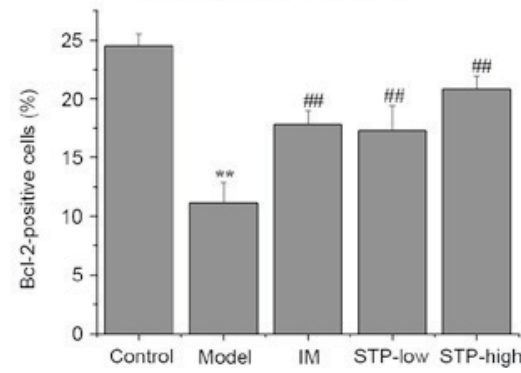

Figure 6. Effects of STP on the protein expression of Bcl-2 in pituitrin-induced acute myocardial ischemia in rat cardiomyocytes. (A) Expression levels of $\mathrm{Bcl}-2$ protein in each group (magnification, $\mathrm{x} 400$ ). Myocardial tissues were processed by IHC staining to detect Bcl-2 expression. (B) Quantification of IHC staining was presented as the percentage of positively-stained cells. Data are presented as the mean + standard deviation, $\mathrm{n}=6$ per group. ${ }^{* *} \mathrm{P}<0.01$ vs. control group; ${ }^{\#} \mathrm{P}<0.01$ vs. model group. STP, Shexiang Tongxin Dropping Pill; IHC, immunohistochemistry; IM, isosorbide mononitrate; STP-low, 20 mg/kg STP group; STP-high, 40 mg/kg STP group.

particularly ST-segment elevation, is the standard method for accurately diagnosing myocardial ischemic injury in animals (18). In the current study, the experimental model group exhibited significant ST-segment elevation following PTT treatment, compared with the control group.

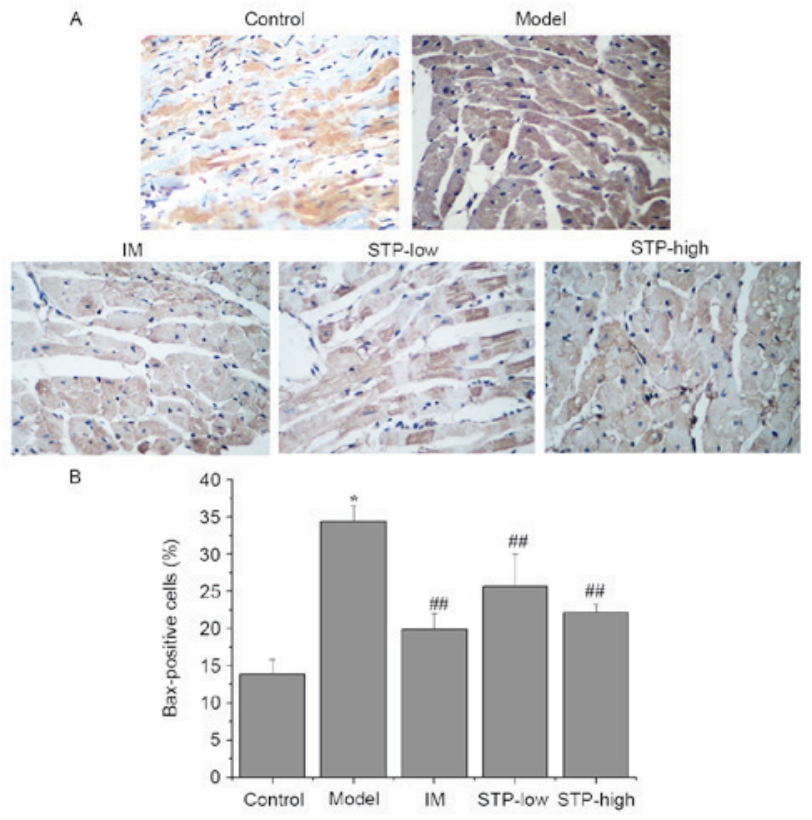

Figure 7. Effects of STP on the protein expression of Bax in pituitrin-induced acute myocardial ischemia in rat cardiomyocytes. (A) Expression levels of Bax protein in each group (magnification, x400). Myocardial tissues were processed by IHC staining to detect Bax expression. (B) Quantification of IHC staining was presented as the percentage of positively-stained cells. Data are presented as the mean + standard deviation, $n=6$ per group. ${ }^{*} \mathrm{P}<0.05$ vs. control group; ${ }^{\# \#} \mathrm{P}<0.01$ vs. model group. STP, Shexiang Tongxin Dropping Pill; Bax, Bcl-2 associated X; IHC, immunohistochemistry; IM, isosorbide mononitrate; STP-low, $20 \mathrm{mg} / \mathrm{kg}$ STP group; STP-high, $40 \mathrm{mg} / \mathrm{kg}$ STP group.

Hemorheological parameters are one of the primary risk factors involved in IHD. Clinical experiments have demonstrated that patients with IHD often exhibited an increase in blood viscosity $(19,20)$. In the present study, STP pretreatment significantly reduced whole blood viscosity in rats following PTT-induced myocardial ischemia. 
LDH and CK-MB are established markers of cellular necrosis, and CK-MB has also been established as a key biomarker of cardiomyocyte injury (21). Serum CK-MB and LDH levels are often employed to monitor the extent of myocardial damage (22). PTT-induced myocardial ischemia resulted in significant leakage of CK-MB and LDH from ruptured myocardial membranes into the serum. This cardiotoxic effect was demonstrated using histopathological examination, which revealed a higher degree of myocardial cell swelling, degeneration and loss of transverse striation in PTT-induced rats. In the current study, pretreatment with STP restored these histopathological changes to a certain degree.

In addition, TUNEL staining in STP pretreatment groups demonstrated decreased cardiomyocyte apoptosis compared with the experimental model group. Furthermore, this myocardial apoptosis may initiate extensive loss of cardiomyocytes, contribute to the pathogenesis of underlying ischemic injury and ultimately result in the deterioration of cardiac function (23).

Cardiomyocyte apoptosis is implicated in various myocardial diseases, including myocardial hypertrophy, heart failure and myocardial ischemia $(24,25)$. Previous studies have demonstrated that a loss of cardiomyocytes as a result of apoptosis is a key factor in myocardial ischemia injury and inevitably leads to heart failure. Cardiomyocyte apoptosis is the predominant form of post-ischemic cardiomyocyte death in the heart and contributes substantially to the impairment of cardiac performance. Reducing cardiomyocyte loss by suppression of cell death is therefore an important strategy in protecting against myocardial diseases $(26,27)$.

During apoptosis, the Bcl-2 family of proteins is the key regulator of mitochondrial-mediated apoptosis. Members of this family include Bcl-2 and Bax, which are anti-apoptotic and proapoptotic proteins, respectively, and have a key role in triggering cell death via the outer mitochondrial membrane $(28,29)$. Typically, the balance between pro- and anti-apoptotic proteins determines whether cells undergo apoptosis or survive under pathophysiological stress following injury $(30,31)$. In the current study, STP pretreatment upregulated the mRNA and protein expression of Bcl-2, while the expression of Bax was downregulated. This indicates that STP may prevent myocardial cells from undergoing apoptosis following ischemic injury, due to its anti-apoptotic and cardioprotective properties.

Additional studies are required to investigate the signaling pathways involved in STP-induced cardioprotection. In addition, the effects and mechanisms of STP on ischemia/reperfusion injury in cultured cardiomyocytes should be investigated.

The present study demonstrated that STP may exert cardioprotective effects on myocardial ischemia by alleviating myocardial ischemic injury and preventing cardiomyocyte apoptosis, based on hemodynamic results, biochemical data, myocardial pathology, and the expression of Bcl-2 and Bax. Therefore, the results of the current study provide evidence that STP may be an effective and promising drug for the clinical prevention and treatment of IHDs.

\section{Acknowledgements}

This study was sponsored by the Foundation of Fujian University of Traditional Chinese Medicine (grant no. X2013026), the
Developmental Fund of Chen Keji Integrative Medicine (grant no. CKJ2013016) and the Education Department of Fujian Province (grant no. JA14163). The authors also thank Inner Mongolia Conba Pharmaceutical Co., Ltd. for their support. Y.Z. is an employee of the Inner Mongolia Conba Pharmaceutical Co., Ltd.

\section{References}

1. Fan J, Li GQ, Liu J, Wang W, Wang M, Qi Y, Xie WX, Liu J, Zhao F, Li Y and Zhao D: Impact of cardiovascular disease deaths on life expectancy in Chinese population. Biomed Environ Sci 27: 162-168, 2014.

2. Sivaraman V and Yellon DM: Pharmacologic therapy that simulates conditioning for cardiac ischemic/reperfusion injury. J Cardiovasc Pharmacol Ther 19: 83-96, 2014.

3. Guo X, Cao W, Yao J, Yuan Y, Hong Y, Wang X and Xing J: Cardioprotective effects of tilianin in rat myocardial ischemia-reperfusion injury. Mol Med Rep 11: 2227-2233, 2015.

4. Panda S, Kar A and Ramamurthy V: Cardioprotective effect of vincristine on isoproterenol-induced myocardial necrosis in rats. Eur J Pharmacol 723: 451-458, 2014.

5. Chen D, Lin S, Xu W, Huang M, Chu J, Xiao F, Lin J and Peng J: Qualitative and quantitative analysis of the major constituents in shexiang tongxin dropping pill by HPLC-Q-TOF-MS/MS and UPLC-QqQ-MS/MS. Molecules 20: 18597-18619, 2015.

6. Xiong M, Jia C, Cui J, Wang P, Du X, Yang Q, Zhu Y, Wang W, Zhang $\mathrm{T}$ and Chen Y: Shexiang Tongxin dropping pill attenuates atherosclerotic lesions in ApoE deficient mouse model. J Ethnopharmacol 159: 84-92, 2015.

7. Hua X, Zhan Y and Li Z: Effect of Shexiang Tongxin Dropping Pill on Cardiac Function in Patients with Chronic Heart Failure. Chin J Integr Med Cardio-/Cerebrovasc Dis, 2011.

8. Ning H: The analysis of Shexiang Tongxin dropping pill on treating coronary heart disease angina pectoris curative effect. Chin J Geriatr Care, 2012.

9. Sui W: Clinical observation of Shexiang Tongxin dropping pill in treating senile unstable agina pectoris. Chin Community Doctors 9: 2, 2011

10. Zhang JX and X; Wang W: Shexiang Tongxin dropping pill Evaluate the Efficacy of the Treatment of Unstable Angina. Chin J Integr Med Cardio-/Cerebrovasc Dis 9: 2, 2011.

11. Liu ZH-QXD-LYLYL-YJJ-GSA-JXX-GWW-M: The Protective Function of Shexiang on Endothelial Injury Induced by AngiotensinIIOsmotic Pump in the Rat. Chin J Clin Med, 2009.

12. Jiang C: Protective Efect of Qi-dan-hua-tan Decoction on Acute Myocardial Ischemia Injury of Rat. J Chengdu Univ Traditional Chin Med, 2011.

13. Liu X, Liu H, Zeng Z, Zhou W, Liu J and He Z: Pharmacokinetics of ligustrazine ethosome patch in rats and anti-myocardial ischemia and anti-ischemic reperfusion injury effect. Int $\mathrm{J}$ Nanomedicine 6: 1391-1398, 2011.

14. Zhang M and Cao H: Research on the Animal Model of Coronary Heart Disease Due to Heart Yang Deficiency. China J Basic Med Traditional Chin Med, 2002.

15. Livak KJ and Schmittgen TD: Analysis of relative gene expression data using real-time quantitative PCR and the 2(-Delta Delta C(T)) Method. Methods 25: 402-408, 2001.

16. Qian Y, Wang S, Xie Y, Wang J, Li H, Zhou X and Liu W: Effect of salvianolic Acid $\mathrm{b}$ and paeonol on blood lipid metabolism and hemorrheology in myocardial ischemia rabbits induced by pituitruin. Int J Mol Sci 11: 3696-3704, 2010.

17. Black JW: Electrocardiographic changes produced in rabbits by vasopressin (pitressin) and their alteration by prolonged treatment with a commercial heart extract. J Pharm Pharmacol 12: 87-94, 1960.

18. Jr JH and Rikkers LF: Success of medical and surgical management of acute variceal hemorrhage. Am J Surg 140: 816-820, 1980.

19. Simpson LO: Angina, ischemic heart disease and blood viscosity. BMJ 339: 464-464, 2015.

20. Sokolov EI, Zykova AA, Sushchik VV and Goncharov IN: Blood viscosity in patients with ischemic heart disease. Kardiologiia 54: 9-14, 2014 (In Russian).

21. Deng C, Sun Z, Tong G, Yi W, Ma L, Zhao B, Cheng L, Zhang J, Cao $F$ and Yi D: $\alpha$-Lipoic acid reduces infarct size and preserves cardiac function in rat myocardial ischemia/reperfusion injury through activation of PI3K/Akt/Nrf2 pathway. PLoS One 8: e58371, 2013. 
22. Gottlieb RA and Engler RL: Apoptosis in myocardial ischemia-reperfusion. Ann N Y Acad Sci 874: 412-426, 1999.

23. Veinot JP, Gattinger DA and Fliss H: Early apoptosis in human myocardial infarcts. Hum Pathol 28: 485-892, 1997.

24. Shen M, Wu RX, Zhao L, Li J, Guo HT, Fan R, Cui Y, Wang YM, Yue SQ and Pei JM: Resveratrol attenuates ischemia/reperfusion injury in neonatal cardiomyocytes and its underlying mechanism. PLoS One 7: e51223, 2012.

25. Song M, Huang L, Zhao G and Song Y: Beneficial effects of a polysaccharide from Salvia miltiorrhiza on myocardial ischemia-reperfusion injury in rats. Carbohydr Polym 98: 1631-1636, 2013.

26. Caroppi P, Sinibaldi F, Fiorucci L and Santucci R: Apoptosis and human diseases: Mitochondrion damage and lethal role of released cytochrome $\mathrm{C}$ as proapoptotic protein. Curr Med Chem 16: 4058-4065, 2009.

27. Foadoddini M, Esmailidehaj M, Mehrani H, Sadraei SH, Golmanesh L, Wahhabaghai H, Valen G and Khoshbaten A: Pretreatment with hyperoxia reduces in vivo infarct size and cel death by apoptosis with an early and delayed phase of protection. Eur J Cardiothorac Surg 39: 233-240, 2011.
28. Wang YL, Wang CY, Zhang BJ and Zhang ZZ: Shenfu injection suppresses apoptosis by regulation of Bcl-2 and caspase-3 during hypoxia/reoxygenation in neonatal rat cardiomyocytes in vitro. Mol Biol Rep 36: 365-370, 2009.

29. Xu J, Min Z, Jian O, Wang J, Zhang Q, Xu Y, Xu Y, Zhang Q, $\mathrm{Xu} \mathrm{X}$ and Zeng $\mathrm{H}$ : Gambogic acid induces mitochondria-dependent apoptosis by modulation of Bcl-2 and Bax in mantle cell lymphoma JeKo-1 cells. Chin J Cancer Res 25: 183-191, 2013.

30. Ji L, Fu FL, Liu W, Cai X, Zhang L, Zheng Q, Zhang H and Gao F: Insulin attenuates myocardial ischemia/reperfusion injury via reducing oxidative/nitrative stress. Am J Physiol Endocrinol Metab 298: E871-E880, 2010.

31. Liao YH, Xia N, Zhou SF, Tang TT, Yan XX, Lv BJ, Nie SF, Wang J, Iwakura Y, Xiao H, et al: Interleukin-17A contributes to myocardial ischemia/reperfusion injury by regulating cardiomyocyte apoptosis and neutrophil infiltration. J Am Coll Cardiol 59: 420-429, 2012. 